\title{
DETERMINANTS OF CONTRACEPTIVE USE DYNAMICS: RESEARCH NEEDS ON DECISION AND CHOICE
}

\author{
AXEL I. MUNDIGO $\dagger$, JAMES F. PHILLIPS $\ddagger$ \\ AND APHICHAT CHAMRATRITHIRONG \\ *Institute for Population and Social Research, Mahidol University, Bangkok, \\ $\dagger$ Special Program of Research in Human Reproduction, World Health \\ Organization, Geneva, $\ddagger$ Research Division, Population Council, New York
}

\begin{abstract}
Summary. Because of the importance of contraceptive behaviour in most societies today a better understanding is needed of the social and behavioural factors affecting contraceptive decisions and choices of individuals and couples. This paper examines the need for longitudinal, theoretically-based studies of contraceptive use dynamics, including the timing, duration and interaction of reproductive events which may be more important than contraceptive technology in the social, cultural and economic context of fertility control. New research methods and appropriate analysis of data are relevant. Consideration of the social context is essential for the formulation and implementation of effective policies relating to the provision of contraceptive services.
\end{abstract}

\section{Introduction}

Understanding the social forces affecting contraceptive use dynamics, and especially those determining reproductive decision and choice, is increasingly necessary for informed population or programme policy formation. Where fertility decline has occurred in developing countries, it has been induced largely by increases in contraceptive use (Bongaarts, 1982; Nortman, 1980; Ross, 1983). But a substantial unmet need for contraception often persists despite readily available and low-cost family planning services. While national policies have contributed to these trends in several settings, strategies for fostering effective and efficient contraceptive practice are often uninformed by social research. Although accessibility of services for providing information and the supply of modern contraception has improved (Tsui, 1985; Jones, 1984), knowledge of methods has become nearly universal (Vaessen, 1980) and adoption has proliferated (Nortman, 1982b), there is often a substantial gap between reproductive intentions and actual contraceptive behaviour (Hollerbach, 1980; Nortman, 1982a; Westoff \& Pebley, 1981). Moreover, levels of ever use are often high where current use prevalence is low (Carrasco, 1981).

To understand the determinants of contraceptive use dynamics social and behavioural studies are required on the mechanisms of decision and choice of 
methods. The study of continuity (including switching and stopping methods) is also important and will be discussed elsewhere. The study of use dynamics includes decision and choice as well as continuity in the practice of contraception and research should be conducted by studying cohorts of women in countries with different levels of programme effort and different environments of choice and service delivery infrastructure. Understanding the determinants of contraceptive choice and adoption as well as continuation is of central policy significance (Mundigo \& Shah, 1988).

Descriptive research has been conducted on contraceptive use dynamics, but general themes and issues that could guide policies on the provision of services are lacking, diffuse, unsystematic and atheoretical. Work has concentrated principally on controlled clinical trials or small-scale introductory trials of contraceptive devices, with little attention to the social determinants of contraceptive behaviour. The effectiveness of contraceptive practice in a population is often variable, in a way that can be largely independent of the intrinsic effectiveness of the methods employed, thus demonstrating that the capacity of contraception to reduce fertility is often as much a function of the characteristics of users as it is of contraceptive technology. This was demonstrated in Taiwan and has been confirmed elsewhere in developing countries (Ross et al., 1972).

In general, research on the determinants of contraceptive use dynamics has concentrated on assessing the effects of reproductive preferences or the stage of couples in their reproductive life cycles. While age, parity and reproductive preferences are of obvious importance in the analysis of continuation rates, there is a need for research on continuity of use by individuals or couples, rather than on devices or modalities, and on the wider factors that explain variance in contraceptive behaviour. Existing services for sustaining contraceptive practice in communities or among couples who are not motivated to use contraception effectively can only be fully used if social and behavioural determinants of contraceptive use dynamics are well understood.

Numerous surveys provide insights into reproductive preferences, contraceptive use attitudes, and levels of former and current contraceptive use. Less information is available on the determinants of decisions in the period following adoption of contraception: the behavioural determinants of method choice, acceptance and continuity of use, switching, and compliance which collectively determine the effectiveness of contraception among couples who have decided to limit or space future pregnancies. Research is therefore needed on the dynamics of contraceptive use, including the timing, duration and interaction of events within the reproductive cycle which relate to the decision and choice, acceptance and continuation, switching and stopping the use of fertility regulation methods. Ideally, longitudinal studies would be guided by specifications of theoretical frameworks that fully consider the dynamics involved. This paper concentrates on behaviour related to contraceptive choice in the study of contraceptive use dynamics.

\section{Decision and choice}

Contraceptive use is initiated through a process of decision and choice that marks a new stage in the reproductive life of couples or individuals and this process is repeated 
at subsequent stages in the reproductive cycle. Post-acceptance decisions are as crucial for understanding use dynamics as are those leading to first-time use: for example, whether to adopt a traditional or modern method, or a spacing method or a terminal option such as sterilization. The process is dynamic, interacting with pregnancy, birth and lactation and affecting the way in which fertility-related events occur. The social forces that determine how contraceptive decision and choice are made in specific circumstances and settings act not only on couples and individuals but also on programmes and services. It is important, therefore, to consider (1) the users and the service providers; (2) health systems: primary health care, clinic services, community-based family planning, commercial outlets, social marketing and (3) contraceptive methods: pill, IUD, condom and other modern as well as traditional methods. For the health system, for example, the questions which relate to choice are: choice of methods actually available; is the system set up to facilitate the individual exercise of choice; do the providers understand the importance of choice as an element in quality of care.

\section{Determinants of choice}

Reproductive health, reproductive rights and quality of care reflect a concern with individual well-being that is achieved through increased decision making, participation and informed choice. But there are also more immediate reasons for studying the determinants of contraceptive choice.

First, there is the relationship between choice and the efficacy of fertility regulation, that is the effect on fertility of the decision to use contraception. While contraceptive methods differ in efficacy, the most important determinant of their effect on fertility is not acceptance but continuity of use, which, in turn, is strongly related to choice. Analysis of the limited information available on social characteristics of acceptors according to method chosen and continuity suggests that method choice is as important as any other user characteristic as a determinant of overall efficacy. This is especially true with regard to traditional versus modern methods. Moreover, it is widely accepted that, by broadening the contraceptive options available to rural couples, contraceptive programmes can enhance service efficiency. Contraceptive preferences, beliefs and needs vary across individuals in all populations, and programmes to increase contraceptive use should therefore offer technologies and services that give the widest possible choice to potential users. It has been observed, for example, in early studies on the use-effectiveness of the IUD, that all method continuation rates are typically substantially higher than first method rates. This implies that multiple contraceptive options among users extend the duration of practice, and that multi-method programmes are intrinsically more effective than single-method ones (Potter, 1971). It is less clear, however, whether increasing available options also affects rates of acceptance. The precise operational and policy implications of method preferences are often unclear. How to implement a user-oriented programme where established service provision practices are governed more by bureaucratic traditions than by users' needs is a challenging issue not widely studied. Research on the efficacy of operational changes designed to improve the availability and acceptability of a wide range of contraceptive options could help to translate findings from social and demographic research into public policy. 
Aggregate data on trends in use after the introduction of new methods suggest that each new method added to a programme can attract new acceptors (Freedman \& Berelson, 1976; Phillips et al., 1989; Ross et al., 1972). No systematic research is available on how new contraceptive options or the accessibility of a wide range of options affects attitudes towards fertility regulation, its overall acceptability or the overall efficacy of service programmes. It has nevertheless been argued that the expansion of choice improves programme credibility and represents a key element in the development of programme support for contraceptive practice (Zeidenstein, 1980).

The second reason for research on the determinants of contraceptive choice derives from the social welfare purposes for establishing contraceptive service programmes. Concern about reproductive rights and reproductive health services that respect individual freedoms necessarily leads to considerations of the importance of maximizing the range of options freely available. Simply offering methods, however, may not expand choice if misperceptions, misinformation, or other social factors constrain choice despite accessibility of multiple method options. It is possible that maximizing choices prevents rumours and generates a more positive climate for decision making that can bring about higher rates of use. A user-oriented programme, focused on establishing an environment conducive to free and informed contraceptive choice, could be more generally acceptable and more widely perceived as serving family welfare, than one emphasizing demographic targets, particular methods or the increase of clinic acceptor loads.

A third reason for studying choice concerns the safety of contraceptive methods, and the fact that the appropriateness of particular methods often differs between individuals. Therefore, the study of contraceptive choice relates closely to concerns about maximizing service quality and the relationships between quality of care and programme effectiveness. Quality of care and client satisfaction can be studied and improved for their own sake, and choice can be viewed as an element of service performance. Many studies on the use-effectiveness of contraception suggest that concerns about side effects are the most frequently cited reasons for terminating use. Whether maximizing choice or improving the quality of information about alternative methods can allay fears about contraception has not been widely studied. Similarly, little is known about services that favour method experimentation for firsttime users, so that women can find out what is the best choice for themselves.

The role of determinants of contraceptive method choice in programme management is also important for understanding the determinants of programme efficiency. The cost effectiveness of each method is different. Policies on the implementation of a multiple versus a single-method approach therefore need to be formulated with a comprehensive understanding of the behavioural and attitudinal aspects of contraceptive method choice. For example, efforts to improve efficiency through special drives or campaigns can have unintended effects which can only be anticipated if careful social research precedes the promulgation of policy (Sullivan $e t$ al., 1976).

\section{Theoretical perspectives}

There has been very little theoretical development in the study of the use dynamics of contraception. Several frameworks to explain the possible causal relationships and 
guide research have been proposed (Phillips, 1981). Given that the immediate determinants of contraceptive decision and choice include such a wide variety of factors, for example, programme, service, method and user-related variables, clarification of the interaction of these variables poses serious difficulties and requires multivariate analytical techniques. Alone, user-related variables include such categories as degree of knowledge of contraceptive options, motivation to reduce or space pregnancies and reproductive intentions. Thus, while no new explanation of these relationships is set out here, these variables are seen as part of a framework in which they are logically and sequentially organized. The causal relationships among these variables are inescapable and present one of the more difficult aspects of this research.

\section{Methodological considerations}

In the study of the determinants of contraceptive choice, a distinction is often drawn between acceptors who seek to space future births and couples who are limiters. Other distinctions may also be required, and it is apparent that research on the determinants of choice behaviour must use either multivariate statistical techniques or microdemographic research methods that examine multiple causal factors. Bulatao \& Palmore (1989) conclude that intensive theoretically guided investigation of the determinants of contraceptive choice might identify policy-relevant findings that cannot be derived from the secondary analysis of existing data. Programme effort variables, including indicators of service quality, the type of providers and programmes, the intensity of programme effort and the availability of alternative methods, are all important determinants of choice. Studies of policies and evaluations of interventions suitable in a particular social and cultural context are also much needed. Health risks (perceived or real) and side effects which may interact with choice behaviour are also important variables. The role of demographic characteristics, including age, fecundity, parity, life cycle, marital status and familial relationships, are also important determinants of contraceptive choice, as are variables reflecting social and economic status and change. These variables, in turn, may relate causally to the demand for children. Desired family size and son preference therefore play a significant role in the commitment to specific fertility regulation alternatives.

Methodologies showing considerable promise for contraceptive choice research are of three general types.

Choice behaviour has been much studied by econometricians. Although contraceptive choice has not been extensively investigated, general economic studies on choice behaviour and household decision-making may be informative for contraceptive choice research (e.g. Akin \& Schwartz, 1988). Statistical methods for the multivariate analysis of discrete alternatives are useful in the analysis of choice behaviour (McFadden, 1973; Judge et al., 1985). Studies of choice behaviour using focus group methods are widely used in marketing, and have been applied to the study of demographic determinants (Schearer, 1981). These methods have not been widely used in research on the determinants of contraceptive choice, but have possible applications.

Methods which have been used extensively in research on reproductive behaviour 
are anthropological micro-demographic studies, which have led to useful demographic theories about fertility determinants, and survey studies, which have provided vital descriptive information on contraceptive behaviour. Future collaborative studies could use large scale statistical data to identify study groups according to specified criteria, and micro-demographic techniques to investigate intensively a limited number of sample sites. While the findings would not be based on statistical analyses, study site selection would be based on the criteria used to specify variance or homogeneity in study sites. The concept of establishing variance between study sites on some key criteria and homogeneity for other selected community characteristics can contribute to the interpretation of micro-demographic research on the determinants of individual behaviour.

Studies of new contraceptives have not adequately considered how new methods affect levels of use, use dynamics, choice or user satisfaction. Moreover, the effect of new contraceptives on service systems and the quality of care is unknown. This lack of scientific knowledge is because contraceptive research has concentrated on modalities rather than users. Experimental cohort studies are needed where designs incorporate matched treatment and comparison areas, and contraceptive behaviour is monitored in populations exposed to a new modality under study.

While secondary analysis of data is appropriate whenever extant data are relevant to a research issue, data collection for research on the determinants of contraceptive choice has not been a priority in the past. Nearly all available studies on this issue are secondary analyses of cross-section survey data. Prospective studies, which involve the prior specifications of a theoretical framework, the design of instruments to investigate the hypotheses presented and thorough sociological or anthropological studies to observe choice behaviour and its determinants over time, are lacking.

\section{Discussion}

Despite over 20 years of research on contraceptive use dynamics, little is known about the social forces that determine whether methods are used effectively or terminated prematurely. Achieving an understanding of the determinants of contraceptive use dynamics, especially of the determinants of decision and choice, is a research issue with important policy implications for service programmes. Use dynamics examines the overall reproductive behaviour of cohorts of women when they are exposed to a variety of contraceptive choices, with the aim of understanding continuation of use, switching patterns and decisions that lead to stopping contraceptive use. Specification of theoretical perspective is needed so that the cross-national relevance of findings for policy is demonstrated. Issues surrounding conceptual arguments and methodological approaches to the study of continuity of use have been left out of this discussion, although the importance of this topic is unquestionable.

Developing a better understanding of the determinants affecting contraceptive behaviour-especially decision and choice-entails resolving challenging methodological problems. Studies, either prospective or retrospective, should collect data on cohorts of users rather than on users of specific methods. If statistical approaches are used, methods are required which permit regression analysis of life table data. Only recently have the relevant methodological tools for such research been demonstrated 
and little progress has yet been achieved in applying these techniques to the study of contraceptive use dynamics. Moreover, micro-demographic research methods have shown considerable promise for developing theories of the determinants of demographic processes. Greater attention to these theoretical concerns and increased application of research methods suited to the examination of the relative effects of multiple causal factors in fertility and contraceptive behaviour are required.

Longitudinal studies of contraceptive use patterns have compared the relative efficacy of contraceptive modalities. In such studies demographic or attitudinal determinants are either potential contaminants of the comparisons of interest, or extraneous factors irrelevant to tests of devices or technologies. In the social, economic and cultural context in which fertility regulation decisions are made, however, technology is often less important than reproductive preferences and the complex interplay of characteristics of families, communities and social and cultural factors.

Policies and programmes aimed at promoting fertility regulation are often introduced in traditional societies where the demand for services is limited and use of contraception has yet to become acceptable. In other contexts, although there is proliferation of the availability of services and information about contraception, the environment of choice is absent. Furthermore, a perceived need for contraception often coexists with widespread misperceptions, fears and concerns about methods. Prevalent health problems can also interact with social and cultural factors to affect profoundly patterns of contraceptive choice and the quality of use. These issues are often compounded by the attitudes, quality of training and method preferences of service providers. Studies on contraceptive use dynamics nevertheless concentrate on ascertaining levels of effective contraceptive use-indicators of prevalence or useeffectiveness, the role of reproductive motives and the stage of eligible couples in their reproductive life cycle as determinants of reproductive behaviour. Greater attention should be given to the determinants of these motives as well as their behavioural consequences so that the attitudes, beliefs and perceptions of fertility regulation can be understood. Such information is particularly useful when delivery systems are changed, especially by the addition of new contraceptive modalities or outreach services. Sound scientific knowledge of the social context in which services must be implemented is a valuable asset for informed policy formulation.

\section{References}

AKIn, J. S. \& Schwartz, B. (1988) The effect of economic factors on contraceptive choice in Jamaica and Thailand: a comparison of mixed multinomial results. Econ. Dev. cult. Change, 36, 503.

BongaAR TS, J. (1982) The fertility inhibiting effects of the intermediate fertility variables. Stud. Fam. Plann. 13, 179.

Bulatao, R. \& Palmore, J. (1989) Choosing a Contraceptive: Factors in Method Choice in Asia and the United States. Westview, Boulder.

Carrasco, E. (1981) Contraceptive Practice.Comparative Studies No. 9. World Fertility Survey, London.

FreEdman, R. \& Berelson, B. (1976) The record of family planning programs. Stud. Fam. Plann. $7,1$. 
Hollerbach, P. (1980) Determinants of the Unmet Need for Family Planning. Pan American Health Organization Report. Population Council, New York.

JonEs, E. F. (1984) The Availability of Contraceptive Services. Comparative Studies No. 37. World Fertility Survey, London.

Judge, G. G., Griffiths, W. E., Hill, R. C., LutkePohl, H. \& Lee, T. C. (1985) Qualitative and limited department variable models. In: The Theory and Practice of Economics, 2nd edn. Wiley, New York.

MCFADDEN, D. (1973) Conditional logit analysis of qualitative choice behavior. In: Frontiers of Economics. Edited by P. Zarembka. Academic Press, New York.

Mundigo, A. \& ShaH, I. (1988) Behavioural and social determinants of fertility regulation. In: Research in Human Reproduction: Biennial Report 1986-1987. Edited by E. Diczfalusy, P. D. Griffin \& J. Khanna. World Health Organization, Geneva.

Nortman, D. (1988) Sterilization and the birth rate. Stud. Fam. Plann. 11, 286.

Nortman, D. (1982a) Population and Family Planning Programs: A Compendium of Data Through 1981. Population Council, New York.

Nortman, D. (1982b) Measuring the unmet need for contraception to space and limit births. Int. Fam. Plann. Perspect. 8, 125.

Phillips, J. F. (1981) A logit regression method for the multivariate analysis of contraceptive attrition. In: The Role of Surveys in the Analysis of Family Planning Programs. Edited by A. I. Hermalin \& B. Entwistle. Ordina, Liège, Belgium.

Phillips, J. F., Hossain, M. B., Hugue, A. A. Z. \& Akbar, J. (1989) A case study of contraceptive introduction: domiciliary DMPA services in rural Bangladesh. In: Demographic and Programmatic Consequences of New Contraceptives. Edited by J. S. Segal, A. O. Tsui \& S. Rogers. Plenum, New York.

PotTER, R. G. (1971) Inadequacy of a one method family planning program. Stud. Fam. Plann. 2 , 1.

Ross, J. A. (1983) Birth control methods and their effects on fertility. In: Determinants of Fertility in Developing Countries, Vol. 2, Fertility Regulation and Institutional Influences. Edited by R. A. Bulatao \& R. D. Lee. Academic Press, New York.

Ross, J. A., Germain, A., Forrest, J. E. \& van Ginneken, J. (1982) Findings from family planning research. Reports on Population/Family Planning, No. 12. Population Council, New York.

SCHEARER, S. B. (1981) The value of focus group research for social action programs. Stud. Fam. Plann. 12, 407.

Sullivan, J., Suyono, H., Bahrawi, W. \& Hartoadi, A. (1976) Contraceptive use-effectiveness in Mojoke Kerto Regency, Indonesia: a comparison of regular program and special drive acceptors. Stud. Fam. Plann. 7, 186.

Tsui, A. O. (1985) Community effects on contraceptive use. In: The Collection and Analysis of Community Data. Edited by J. Casterline. International Statistical Institute, Voorburg, Netherlands.

VAEssen, M. (1980) Knowledge of Contraceptive Methods. Comparative Studies No. 8. World Fertility Survey, London.

Westoff, C. F. \& Pebley, A. R. (1981) Alternative measures of unmet need for family planning. Int. Fam. Plann. Perspect. 7, 126.

ZEIDENSTEIN, G. (1980) The user perspective: an evolutionary step in contraceptive service programs. Stud. Fam. Plann. 11, 24. 